\title{
Alpha-Defensin versus Leukocyte Esterase in Periprosthetic Joint Infection: An Updated Meta-Analysis
}

\author{
Zhizhuo Li, ${ }^{1}$ Qingyu Zhang, ${ }^{2}$ Lijun Shi, ${ }^{3}$ Fuqiang Gao, ${ }^{4}$ Wei Sun $\mathbb{D}^{4},{ }^{4}$ and Zirong Li $^{4}$ \\ ${ }^{1}$ Department of Orthopedics, Peking University China-Japan Friendship School of Clinical Medicine, 2 Yinghuadong Road, \\ Chaoyang District, Beijing 100029, China \\ ${ }^{2}$ Department of Orthopedics, Shandong Provincial Hospital Affiliated to Shandong University, No. 324, Road Jing Wu Wei Qi, Jinan, \\ 250021 Shandong, China \\ ${ }^{3}$ Department of Orthopedics, Graduate School of Peking Union Medical College, China-Japan Friendship Institute of \\ Clinical Medicine, 2 Yinghuadong Road, Chaoyang District, Beijing 100029, China \\ ${ }^{4}$ Beijing Key Laboratory of Immune Inflammatory Disease, China-Japan Friendship Hospital, 2 Yinghuadong Road, \\ Chaoyang District, Beijing 100029, China
}

Correspondence should be addressed to Wei Sun; cjfhsunw@163.com

Received 29 July 2020; Revised 22 September 2020; Accepted 26 October 2020; Published 18 November 2020

Academic Editor: Haining Zhang

Copyright (C) 2020 Zhizhuo Li et al. This is an open access article distributed under the Creative Commons Attribution License, which permits unrestricted use, distribution, and reproduction in any medium, provided the original work is properly cited.

Periprosthetic joint infection (PJI) is a devastating complication after arthroplasty. Prompt establishment of an infection diagnosis is critical but can be very challenging at present. In order to evaluate the diagnostic accuracy of alpha-defensin or leukocyte esterase for PJI, we performed systematic research in PubMed, Embase, and Cochrane Library to retrieve relevant studies. Data extraction and quality assessment were performed by two reviewers independently. A total of thirty-one eligible studies were finally included in the quantitative analysis. The pooled sensitivity and specificity of alpha-defensin ( 21 studies) for the diagnosis of PJI were 0.89 ( $95 \%$ confidence interval (CI), 0.83 to 0.93 ) and 0.96 (95\% CI, 0.95 to 0.97 ), respectively. The value of the pooled diagnostic odds ratios (DOR) of alpha-defensin for PJI was 209.14 (95\% CI, 97.31 to 449.50), and the area under the curve (AUC) was 0.98 (95\% CI, 0.96 to 0.99 ). The pooled sensitivity and specificity of leukocyte esterase (17 studies) for the diagnosis of PJI were 0.90 (95\% CI, 0.84 to 0.95 ) and 0.96 (95\% CI, 0.93 to 0.97), respectively. The value of the DOR of leukocyte esterase for PJI was 203.23 (95\% CI, 96.14 to 429.61 ), and the AUC was 0.98 (95\% CI, 0.96 to 0.99). Based on the results of our meta-analysis, we can conclude that alphadefensin and leukocyte esterase are valuable synovial fluid markers for identifying PJI with comparable high diagnostic accuracy.

\section{Introduction}

Periprosthetic joint infection (PJI) occurs in more than $2 \%$ of patients undergoing joint arthroplasty, acting as the leading cause of total knee arthroplasty failure and the third most common indication for hip revision [1-3]. Meanwhile, PJI imposes a heavy financial burden on patients and the healthcare system. The cost of using a debridement-and-retention protocol and one-stage revision to treat a single PJI is approximately 3-fold and 3.4-fold the cost of primary implantation, respectively [4]. There are some methods in use, such as perioperative antibiotics, antibiotic-impregnated bone cement, and antimicrobial-loaded implants, which can reduce the incidence of PJI but cannot completely eliminate it [5]. A timely diagnosis of an infection may be helpful in eliminating lesions completely and thus avoiding radical treatments such as one-stage or two-stage revision [6].

The most common clinical manifestations of PJI are pain, induration or edema, wound drainage or effusion, surgical site erythema in the early stage, and sinus in the later stage. Conventionally, the most used diagnostic algorithms for the diagnosis of PJI are peripheral blood tests, imaging examination, and microbiological examination. No single test is accurate enough for PJI diagnosis, and the test results must be combined with clinical history and symptoms; however, symptoms of PJI are usually nonspecific in the early stage. Groups such as the American Academy of Orthopedic Surgeons (AAOS), Infectious Diseases Society of America 
(IDSA), and the Musculoskeletal Infection Society (MSIS) have proposed several criteria for the diagnosis of PJI [7-9], but applications of these definitions are limited in daily clinical practice due to their complexity. A more specific and sensitive routine test for the diagnosis of PJI is therefore badly needed.

Alpha-defensin and leukocyte esterase are both secreted by activated neutrophils in the presence of pathogenic infection. As an antimicrobial peptide, alpha-defensin binds to and destroys invading pathogens [10] while leukocyte esterase has been widely used as an indicator for the assessment of urinary tract infections in the clinic [11]. The content of alpha-defensin is normally detected by lateral flow test [12] or laboratory-based alpha-defensin immunoassay [13] and that of leukocyte esterase by colorimetric strip test [14]. Synovial fluid alpha-defensin and leukocyte esterase were first proposed as novel diagnostic biomarkers for PJI, respectively, by Deirmengian et al. in 2014 [15] and Parvizi et al. in 2011 [16], but their diagnostic efficacy has yet to be confirmed.

In 2016, Wyatt et al. [17] conducted a meta-analysis of the usefulness of alpha-defensin and leukocyte esterase as diagnostic tools for PJI, with pooled sensitivity and specificity being 1.00 (95\% confidence interval (CI), 0.82 to 1.00 ) and 0.96 (95\% CI, 0.89 to 0.99 ), respectively, for alphadefensin, and 0.81 (95\% CI, 0.49 to 0.95$)$ and $0.97(95 \%$ CI, 0.82 to 0.99 ), respectively, for leukocyte esterase. However, in this systematic analysis [17], only 11 studies were included and four of six studies concerning alpha-defensin came from the same research group, which might hinder the generalization of their results. Additionally, the value of pooled sensitivity of leukocyte esterase was quite different from that reported in the literature published in recent years. Moreover, heterogeneity tests and subgroup analysis were not conducted by Wyatt et al. [17]. We carried out this updated meta-analysis to further assess the diagnostic accuracy of alpha-defensin and leukocyte esterase for periprosthetic joint infection.

\section{Materials and Methods}

The methodological approach to evidence searching and synthesis described in this article was based on the Cochrane Collaboration's diagnostic test accuracy method [18]. We carried out the current systematic review and reported the findings in accordance with the standards of the Preferred Reporting Items for Systematic Reviews and Meta-Analyses (PRISMA) [19]. No ethical approval or informed consent was required for this article because all data were retrieved from published literature. Searching for studies, identification of eligibility, data extraction, and quality assessment were performed by two investigators (ZZ Li and QY Zhang) independently. Any disagreement was resolved through discussion, and the two researchers had to come to a consensus.

2.1. Search Strategy. Three electronic databases PubMed, Embase, and Cochrane Library were searched on June 18, 2020, and no time limitation was applied. Vocabulary and syntax were specifically adapted according to the database.
We used "periprosthetic joint infection" as our diagnosis of interest and "leukocyte esterase" or "alpha defensin" as our target index, and the full search strategy is shown in Table 1. No language limitation was applied. Reference lists of relevant articles were also screened manually for any additional possible records.

2.2. Inclusion Criteria. Studies included in the systematic review needed to meet the following criteria: (1) patients who had undergone joint replacement, (2) sufficient synovial fluid had been aspirated to meet the needs of the tests, (3) the leukocyte esterase or alpha-defensin tests were performed on the synovial fluid, (4) the diagnosis of PJI was confirmed by the MSIS, AAOS, and IDSA guidelines or utilizing a combination of clinical data, which must include microbiological examination, (5) either a prospective or a retrospective study design, and (6) sufficient data could be extracted to construct a $2 \times 2$ contingency table. If more than one study provided overlapping data, only the most comprehensive or latest one was included. Case reports, commentaries, expert opinion, and narrative reviews were excluded.

2.3. Data Extraction and Quality Assessment. Requisite data extracted and recorded to standardized excel files included surname of the first author, publication year, study inclusion interval, country, study design, demographic information of participants, number of infected/total joints, site of arthroplasty, cut-off value, method used to assess alpha-defensin and leukocyte esterase, standard reference, and number of false/true positive and false/true negative cases. The methodological quality of included studies was appraised according to the QUADAS- (Quality Assessment of Diagnostic Accuracy Studies-) 2 tool, which consists of four key domains (i.e., patient selection, index test, reference standard, and flow and timing). Risk of bias was assessed in each domain, and concerns about applicability were assessed in the first three domains with signaling questions. These questions were answered with "yes" for a low risk of bias/concerns, "no" for a high risk of bias/concerns, or "unclear" when relevant information was not clearly provided [20].

2.4. Statistical Analyses. Pooled sensitivity, specificity, positive likelihood ratio (PLR), negative likelihood ratio (NLR), and diagnostic odds ratio (DOR) were calculated using the bivariate meta-analysis framework. The bivariate model employs a random-effects approach, and the statistical properties of the bivariate model are suited to performing diagnostic meta-analyses. In addition, summarized receiver operating characteristic (sROC) curves were constructed, with the area under the curve (AUC) depicting the accuracy of tests. Heterogeneity among included studies was assessed using the $I^{2}$ statistic. An $I^{2}$ value of $0 \%$ implied no observed heterogeneity, and values of $>50 \%$ indicated substantial heterogeneity. For studies with substantial heterogeneity, the Spearman correlation coefficient was calculated to determine whether a threshold effect existed and we also performed metaregression analyses to find the source of variability. Subgroup analyses based on covariates were also performed to ascertain the stability of results. 
TABLE 1: Search strategy.

\begin{tabular}{ll}
\hline Alpha-defensin search & Leukocyte esterase search \\
\hline (1) Alpha-defensins or alpha defensins & (1) Leukocyte esterase or leucocyte esterase \\
(2) Neutrophil antimicrobial peptides or antimicrobial peptides, & (2) Leukocyte indoxyl esterase or indoxyl esterase \\
neutrophil or peptides, neutrophil antimicrobial & (3) Arthroplasty or joint prosthesis \\
(3) Arthroplasty or joint prosthesis or joint replacement & or joint replacement \\
(4) Periprosthetic infection or prosthetic joint infection or & (4) Periprosthetic infection or prosthetic joint \\
prosthesis-related infections & infection or prosthesis-related infections \\
(5) Bacterial infections or surgical wound infection & (5) Bacterial infections or surgical wound infection \\
(6) 1 or 2 & (6) 1 or 2 \\
(7) 4 or 5 & (7) 4 or 5 \\
(8) 3 and 7 & (8) 3 and 7 \\
(9) 6 and 8 & (9) 6 and 8 \\
\hline
\end{tabular}

Database: PubMed, Embase, and Cochrane Library. Other databases: subject-specific databases, dissertation databases, or grey literature databases.

The value of a two-sided $P<0.05$ was considered statistically significant in all statistical tests. Stata version 13 (StataCorp, College Station, TX, USA) was used to analyze data from the included studies, and Review Manager Software version 5.3 (Cochrane Collaboration, Oxford, UK) was used to assess the methodological quality of included studies.

\section{Results}

3.1. Search Results and Study Selection. A total of 1356 records were identified by searching databases and removing duplicates. After the initial screening of titles and abstracts, 59 articles were further assessed by scrutinizing the full texts against the predesigned criteria, and eventually, 35 articles $[12,14-16,21-51]$ were included in quantitative analysis. Selection processes for eligible studies are depicted in Figure 1.

3.2. Study Characteristics. Twenty-three studies [12, 16, 21, $22,25,27-39,41-44,47]$ were prospective, and twelve studies $[14,15,23,24,26,40,45,46,48-51]$ were retrospective. Thirty-four studies $[12,15,16,21-51]$ were cohort studies whereas only one study [14] was a case-control study. Twenty-one studies involving a total of 1928 patients (650 joints with PJI) explored the diagnostic accuracy of alpha-defensin $[12,14,15,23-27,29,30,33-35,37,38$, $41,45,46,48-50]$, among which eight studies [12, 27, 29, $30,34,35,37,48]$ used a lateral flow test to assess alphadefensin, twelve $[14,15,24-26,33,38,41,45,46,49,50]$ adopted a laboratory-based alpha-defensin immunoassay, and the testing methods used were not reported in one studies [23]. The mean ages of included patients ranged from 61.7 to 71.0 years, and the proportion of males ranged from $40.3 \%$ to $61.5 \%$.

Meanwhile, seventeen studies involving a total of 1963 patients (571 joints with PJI) explored the diagnostic accuracy of leukocyte esterase for PJI $[14,16,21,22,26,28,31$, $32,36,39,40,42-44,47,50,51]$. All studies used the standard chemical test strip, among which two studies $[16,32]$ used an automated reader to define the final result while thirteen studies $[14,21,22,26,28,31,36,39,40,42-44,47,50,51]$ used the naked eye. The mean ages of included patients ranged from 60.3 to 71.0 years, and the proportion of males ranged from $21.7 \%$ to $60.9 \%$.

Eventually, 38 datasets were available for the appraisal of diagnostic accuracy of alpha-defensin and leukocyte esterase for PJI. The main characteristics of included studies are summarized in Table 2.

3.3. Results of Quality Assessment. The results of QUADAS2 assessments for each included study are shown in Figure 2. In each key domain, the proportion of high risk was less than 5\%, which indicated that the quality of included studies was good.

3.4. Diagnostic Value of Alpha-Defensin for PJI. As shown in Figure 3, the pooled sensitivity and specificity of alphadefensin for diagnosing PJI were 0.89 (95\% CI, 0.83 to 0.93 ) and 0.96 (95\% CI, 0.95 to 0.97$)$, respectively. The pooled PLR, NLR, and DOR were 23.18 (95\% CI, 15.79 to 34.03 ), 0.11 (95\% CI, 0.07 to 0.18 ), and 209.14 (95\% CI, 97.31 to 449.50), respectively. The AUC of alpha-defensin for PJI was 0.98 (95\% CI, 0.96 to 0.99 ) (Figure 4). The $I^{2}$ statistics for sensitivity and specificity values were $64.09 \%$ (95\% CI, $47.36 \%$ to $80.82 \%)$ and $51.82 \%(95 \% \mathrm{CI}, 27.77 \%$ to $75.87 \%$ ), respectively, which indicated substantial heterogeneity among included studies. We subsequently performed subgroup and metaregression analysis to explore the source of heterogeneity. The summary data of alpha-defensin for PJI calculated using STATA and estimation of the Spearman correlation coefficient $(P$ value $<0.01)$ indicated that the proportion of heterogeneity was likely due to the threshold effect. In addition, for the nonthreshold effect, we also performed metaregression analysis. Age, patient sample size, study design, and method of testing were used as covariates. The results of metaregression analysis (Figure 5) revealed that patient sample size and method of testing accounted for the heterogeneity of sensitivity and specificity while study design accounted for the heterogeneity of specificity.

Subgroup analyses were performed according to the testing method (lateral flow test or laboratory-based alphadefensin immunoassay), sample size $(<100$ or $\geq 100)$, and study design (retrospective or prospective), and the pooled results are presented in Table 3 . 


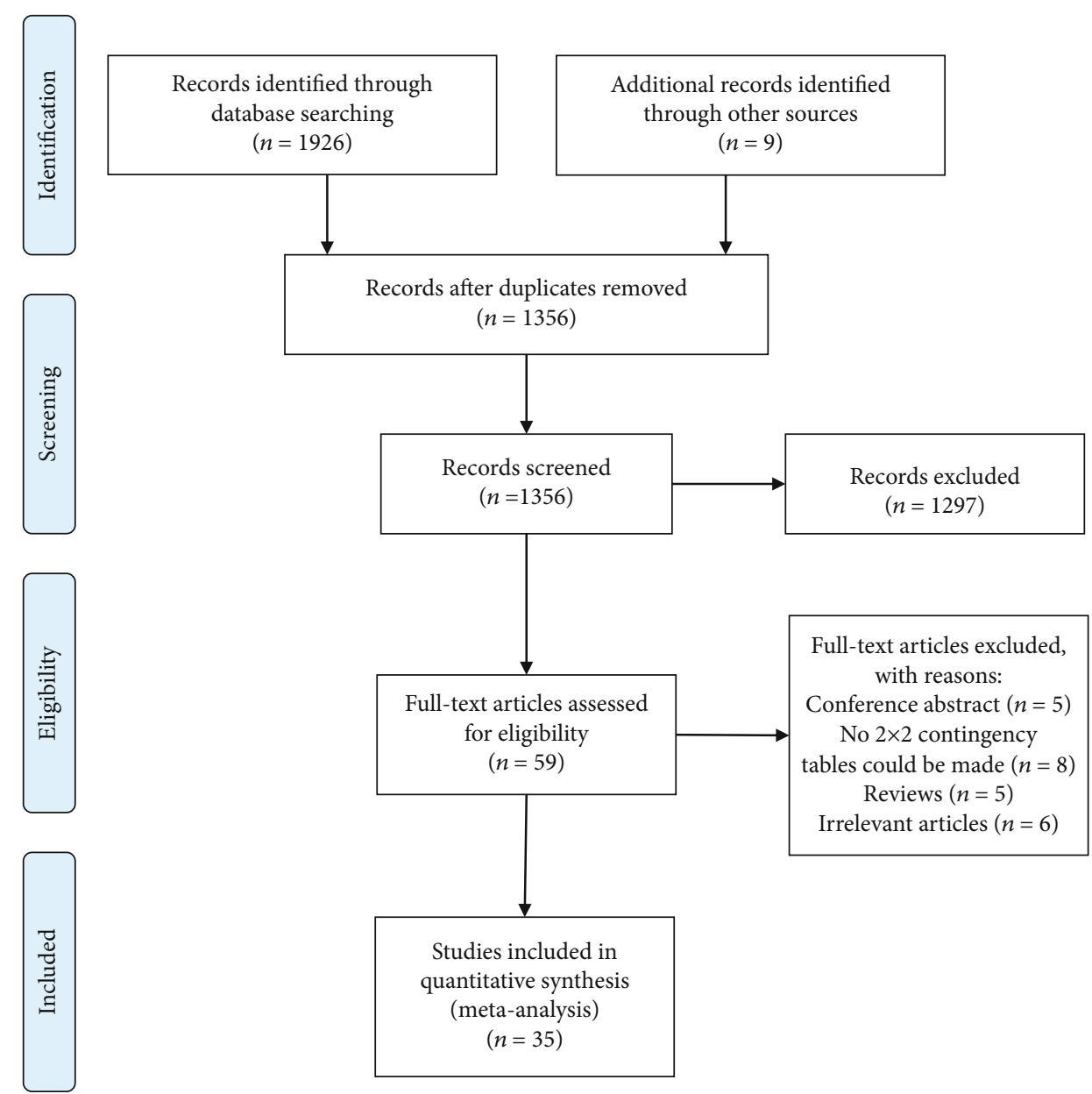

Figure 1: Selection process of included studies.

3.5. Diagnostic Value of Leukocyte Esterase for PJI. As shown in Figure 3, the pooled sensitivity and specificity of leukocyte esterase for diagnosing PJI were 0.90 (95\% CI, 0.84 to 0.95 ) and 0.96 (95\% CI, 0.93 to 0.97 ), respectively. The pooled PLR, NLR, and DOR were 20.25 (95\% CI, 13.71 to 29.90), 0.10 (95\% CI, 0.06 to 0.18 ), and 203.23 (95\% CI, 96.14 to 429.61), respectively. The AUC of leukocyte esterase for PJI was 0.98 (95\% CI, 0.96 to 0.99) (Figure 4). The $I^{2}$ statistics for sensitivity and specificity values were $78.63 \%$ (95\% CI, $68.95 \%$ to $88.32 \%)$ and $58.12 \%$ (95\% CI, $35.60 \%$ to $80.63 \%$ ), respectively, which indicated substantial heterogeneity among the included studies. The summary data of leukocyte esterase for PJI calculated using STATA and estimation of the Spearman correlation coefficient $(P$ value $>0.05)$ indicated the absence of a threshold effect. In addition, for the nonthreshold effect, we also performed metaregression analysis with age, patient sample size, study design, and cut-off used as covariates. The results of metaregression analysis (Figure 5) revealed that patient sample size, study design, and cut-off accounted for the heterogeneity of specificity.

Among the 17 studies, subgroups were divided according to cut-off value $(++$ or $++/+)$ and patient sample size $(<100$ or $\geq 100)$, and the pooled results are presented in Table 3 .

\section{Discussion}

Accurate and fast diagnosis of periprosthetic infections remains a challenging problem. The present "gold standard" definition for PJI proposed by the Musculoskeletal Infection Society (MSIS) is adopted by most physicians, which requires accordance with either of two major criteria (sinus tract communication with the prosthesis or a pathogen isolated by culture from at least two separate tissue or fluid samples) or four of six minor criteria (elevated erythrocyte sedimentation rate/ESR, C-reactive protein/CRP, white blood cell/WBC count, and percentage of polymorphonuclear leukocytes/PMN; the presence of purulence; isolation of a microorganism in one culture; and greater than five neutrophils per high-power field) [9]. Our results revealed that alphadefensin and leukocyte esterase were highly sensitive and specific in identifying PJI (pooled sensitivity of 0.89 and 0.90 , respectively, with pooled specificity of 0.96 and 0.96 , respectively). We also estimated alpha-defensin and leukocyte esterase combined in the diagnosis of PJI. The corresponding sensitivity and specificity of alpha-defensin associated with leukocyte esterase test's parallel and serial test were $0.989,0.9216$ and $0.801,0.9984$, respectively. The values of AUC for alpha-defensin and leukocyte esterase were both 


\begin{tabular}{|c|c|c|c|c|c|c|c|c|c|c|c|c|c|c|c|c|c|c|}
\hline 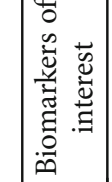 & \& & 党 & 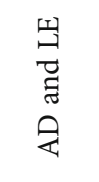 & 安 & 是 & 完 & 是 & 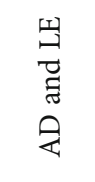 & 安 & 完 & 安 & 完 & 安 & 安 & 宗 & 安 & 安 & 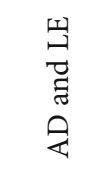 \\
\hline 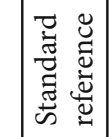 & $\stackrel{n}{\Sigma}$ & $\stackrel{\tilde{n}}{\Sigma}$ & $\sum_{\Sigma}^{\tilde{n}}$ & $\stackrel{\tilde{n}}{\Sigma}$ & $\frac{\tilde{\omega}}{\sum}$ & $\tilde{n}$ & $\sum_{\Sigma}^{\infty}$ & $\frac{\tilde{n}}{\tilde{n}^{2}}$ & $\stackrel{n}{\Sigma}$ & $\stackrel{\infty}{\Sigma}$ & $\tilde{n}$ & $\stackrel{\tilde{N}}{\Sigma}$ & $\stackrel{n}{\Sigma}$ & $\frac{n}{\Sigma}$ & $\stackrel{\mathscr{G}}{\Sigma}$ & $\tilde{n}$ & 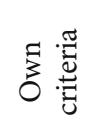 & $\sum_{\Sigma}^{\mathscr{n}}$ \\
\hline 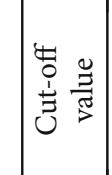 & 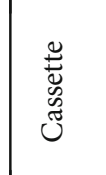 & 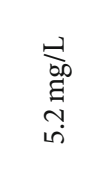 & 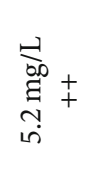 & 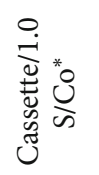 & 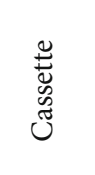 & $\overleftrightarrow{z}$ & 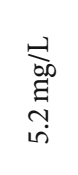 & 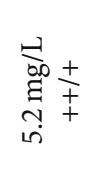 & 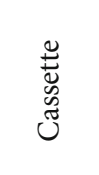 & $\begin{array}{l}\tilde{\Xi} \\
\tilde{\Xi} \\
\tilde{D} \\
\tilde{J}\end{array}$ & 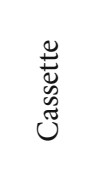 & $\begin{array}{l}00 \\
0 \\
5 \\
0 \\
0\end{array}$ & $\begin{array}{l}\mathscr{\Xi} \\
\tilde{\Xi} \\
\tilde{J} \\
\tilde{J}\end{array}$ & 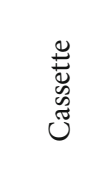 & 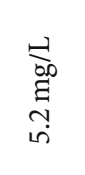 & 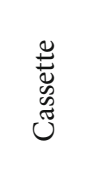 & $\overleftrightarrow{z}$ & 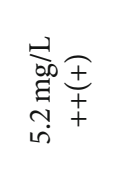 \\
\hline 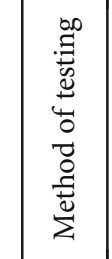 & 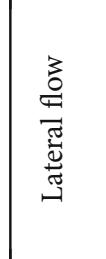 & 芯 & 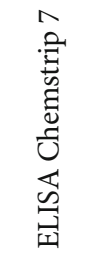 & 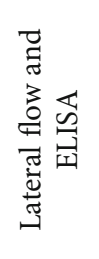 & 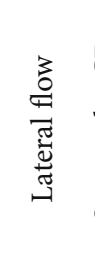 & 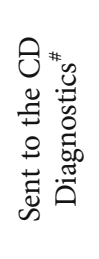 & $\begin{array}{l}\overparen{心} \\
\stackrel{\leftrightarrows}{\mid c}\end{array}$ & 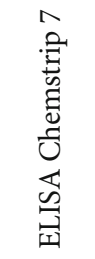 & 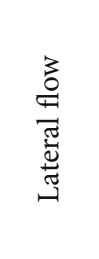 & 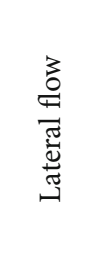 & 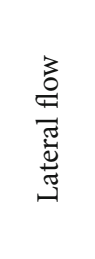 & 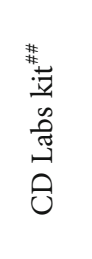 & 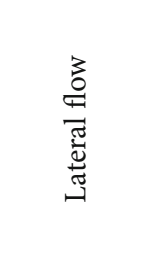 & 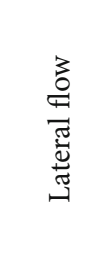 & 芯 & 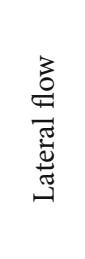 & 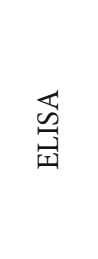 & 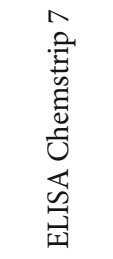 \\
\hline 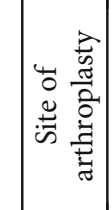 & $\overleftrightarrow{Z}$ & $\begin{array}{l}\curvearrowright \cong \\
\ddot{\otimes} \Xi \\
\tilde{\ddot{g}}\end{array}$ & 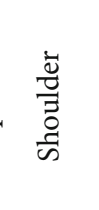 & 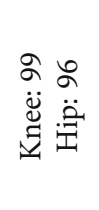 & 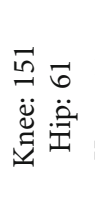 & 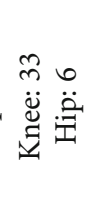 & 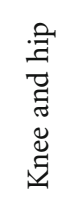 & 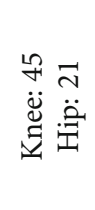 & 总 & 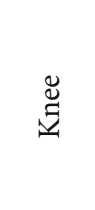 & 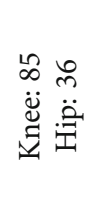 & 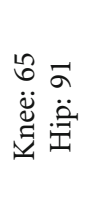 & 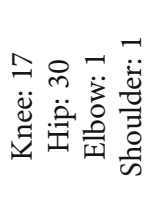 & $\begin{array}{l}a= \\
\ddot{\ddot{\otimes}}= \\
\ddot{\Xi}\end{array}$ & 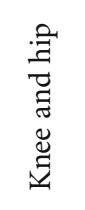 & 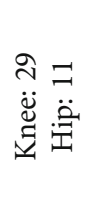 & 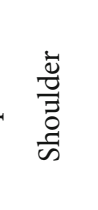 & 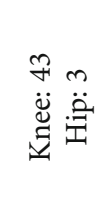 \\
\hline 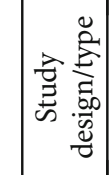 & $\simeq \frac{\overrightarrow{0}}{0}$ & $\simeq \frac{\overrightarrow{0}}{0}$ & $\simeq \frac{\overrightarrow{0}}{0}$ & $a \frac{\overrightarrow{0}}{0}$ & $2 \frac{\overrightarrow{0}}{0}$ & $\simeq \frac{\overrightarrow{0}}{0}$ & $\simeq \frac{\overline{0}}{\frac{\pi}{0}}$ & $\simeq \frac{\overrightarrow{0}}{0}$ & $a \frac{\overrightarrow{0}}{0}$ & a $\frac{\overrightarrow{0}}{0}$ & $a \frac{\overrightarrow{0}}{0}$ & $a \frac{5}{0}$ & a $\frac{5}{0}$ & a & $n \frac{\overline{0}}{0}$ & a $\frac{5}{0}$ & $0 \frac{5}{0}$ & 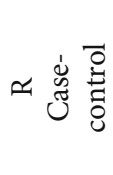 \\
\hline 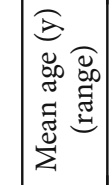 & $\hat{\sigma}$ & 艺 & $\begin{array}{l}0 \\
\infty \\
0 \\
b \\
0 \\
0 \\
0\end{array}$ & 㞱 & 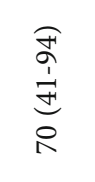 & $\begin{array}{l}\widehat{\infty} \\
\infty \\
1 \\
\infty \\
0 \\
\beta\end{array}$ & $\bullet$ & $\begin{array}{l}\widehat{n} \\
\hat{1} \\
\hat{0} \\
\infty \\
0\end{array}$ & $\begin{array}{l}\hat{\alpha} \\
\text { o } \\
\dot{b}\end{array}$ & 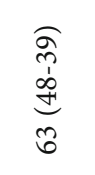 & 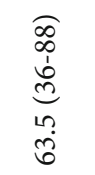 & 艺 & $\begin{array}{l}a \\
\infty \\
\dot{d} \\
d \\
0 \\
0\end{array}$ & $\begin{array}{l}\widehat{\infty} \\
\infty \\
0 \\
\hat{d} \\
\hat{0} \\
\hat{0}\end{array}$ & $\begin{array}{l}\stackrel{9}{7} \\
+1 \\
0 \\
0 \\
0\end{array}$ & 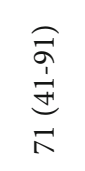 & $\begin{array}{l}\stackrel{+}{i} \\
\underset{7}{+1} \\
\stackrel{+}{0} \\
\stackrel{0}{0}\end{array}$ & $\frac{\widehat{0}}{\hat{\sigma}}$ \\
\hline 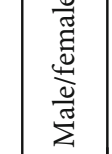 & $\overleftrightarrow{Z}$ & $\underset{\text { a }}{\stackrel{a}{\sigma}}$ & $\underset{\substack{0 \\
\infty}}{\stackrel{\infty}{n}}$ & $\underset{⿱}{\stackrel{\Xi}{E}}$ & $\stackrel{8}{\stackrel{1}{0}}$ & $\overleftrightarrow{Z}$ & $\overleftrightarrow{Z}$ & 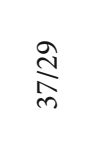 & $\underset{\sim}{\stackrel{n}{\not}}$ & 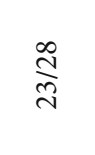 & $\overleftrightarrow{Z}$ & $\frac{8}{8}$ & 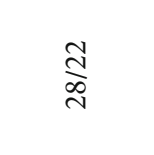 & $\underset{\Xi}{\Xi}$ & $\frac{\Re}{\frac{n}{2}}$ & $\overleftrightarrow{z}$ & $\underset{\vec{g}}{\stackrel{N}{\sigma}}$ & $\underset{\infty}{\infty}$ \\
\hline 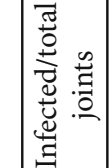 & $\frac{\vec{n}}{2}$ & 灾 & 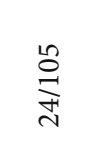 & 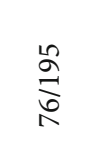 & 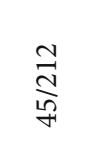 & 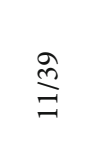 & $\stackrel{P}{\stackrel{P}{m}}$ & 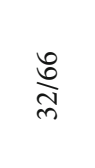 & $\vec{F}$ & $\frac{\vec{n}}{6}$ & $\underset{\stackrel{J}{J}}{\vec{J}}$ & $\underset{\stackrel{2}{2}}{\stackrel{2}{\pi}}$ & $\frac{g}{g}$ & 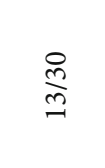 & 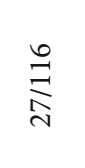 & 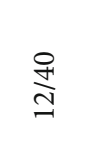 & $\stackrel{m}{\equiv}$ & $\frac{\substack{1 \\
\sim}}{\sim}$ \\
\hline 吾 & 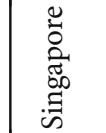 & 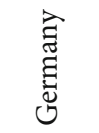 & 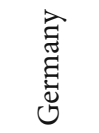 & 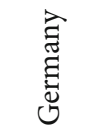 & 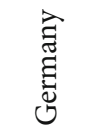 & $\begin{array}{l}\overleftrightarrow{s} \\
\widetilde{D}\end{array}$ & 岕 & 公 & 䔍 & 预 & 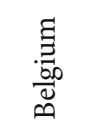 & 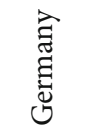 & 莺 & 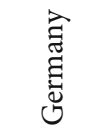 & 岕 & 悉 & 岕 & 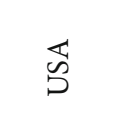 \\
\hline 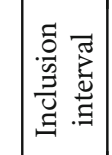 & 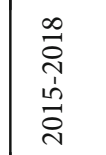 & $\begin{array}{l}\infty \\
\stackrel{1}{1} \\
\stackrel{1}{0} \\
\stackrel{\text { ते }}{ }\end{array}$ & 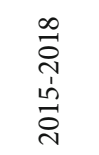 & 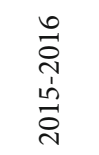 & 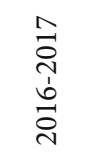 & 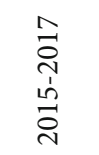 & $\begin{array}{l}0 \\
\stackrel{1}{1} \\
\text { I } \\
\stackrel{i}{1}\end{array}$ & 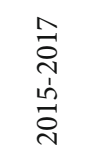 & 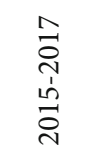 & 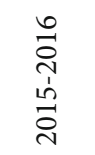 & $\begin{array}{l}0 \\
\stackrel{7}{1} \\
\omega \\
\stackrel{\sim}{a}\end{array}$ & 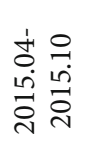 & 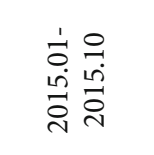 & 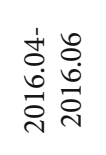 & 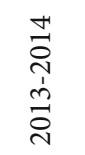 & $\begin{array}{l}n \\
\stackrel{n}{0} \\
\text { I } \\
\dot{I} \\
\stackrel{4}{1}\end{array}$ & $\begin{array}{l}m \\
\stackrel{n}{\sim} \\
\stackrel{\sim}{\sim} \\
\stackrel{\sim}{\sim}\end{array}$ & 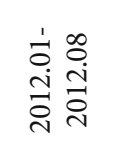 \\
\hline 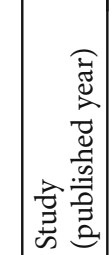 & 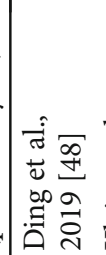 & 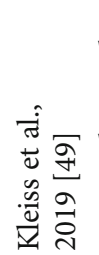 & 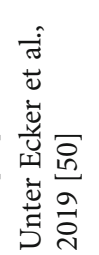 & 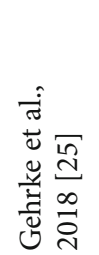 & 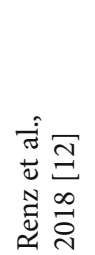 & 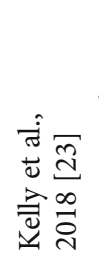 & 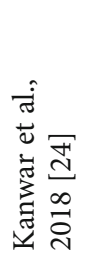 & 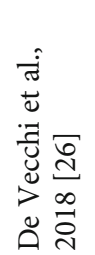 & 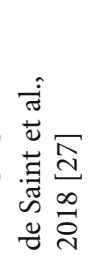 & 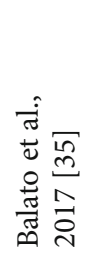 & 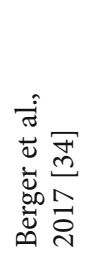 & 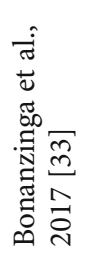 & 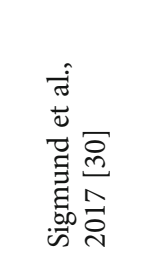 & 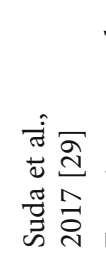 & 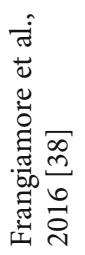 & 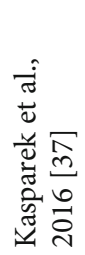 & 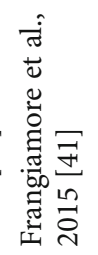 & 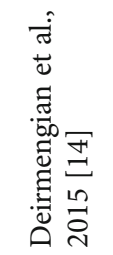 \\
\hline
\end{tabular}




\begin{tabular}{|c|c|c|c|c|c|c|c|c|c|c|c|c|c|c|c|c|c|c|}
\hline 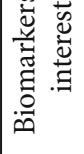 & 完 & 安 & 安 & 덥 & 덥 & 孚 & 䄸 & 덥 & 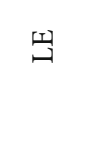 & 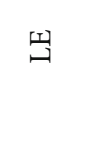 & 岏 & 嵒 & 凷 & 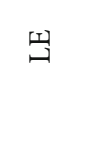 & 牙 & 법 & 牙 & 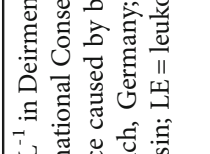 \\
\hline 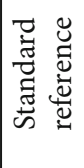 & 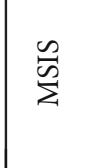 & 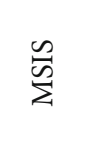 & $\stackrel{n}{n}$ & $\sum_{\Sigma}^{n}$ & $\sum_{\Sigma}^{n}$ & $\sum_{n}^{n}$ & $\sum_{\Sigma}^{n}$ & $\sum_{\Sigma}^{n}$ & $\sum_{\Sigma}^{2}$ & $\stackrel{n}{\tilde{n}}$ & $\sum_{\Sigma}^{n}$ & 芯 & 娄 & $\stackrel{\tilde{n}}{\sum^{n}}$ & $\tilde{n}$ & $\tilde{n}_{\Sigma}^{\infty}$ & $\stackrel{n}{n}$ & 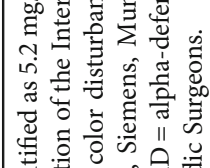 \\
\hline 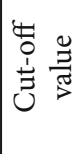 & 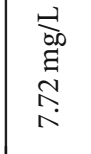 & 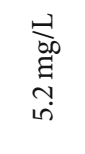 & $\begin{array}{l}\underset{00}{00} \\
\underset{0}{\infty} \\
+\end{array}$ & $\stackrel{+}{+}$ & $\stackrel{\substack{+\infty \\
+\\
+}}{+}$ & $\stackrel{+}{+}$ & 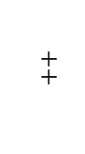 & + & $\stackrel{\substack{ \pm++}}{+}$ & $\stackrel{+}{+}$ & $\underset{+}{+}$ & $\stackrel{+}{+}$ & $\stackrel{+}{+}$ & $\ddagger$ & 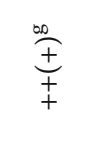 & $\stackrel{+}{+}$ & $\frac{\stackrel{00}{+}}{+}$ & 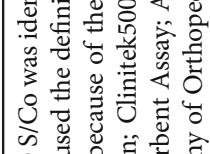 \\
\hline 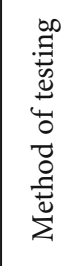 & 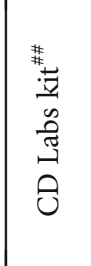 & 荡 & 茫 & 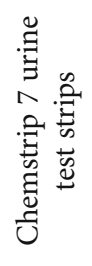 & 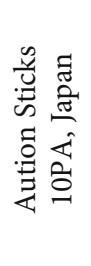 & 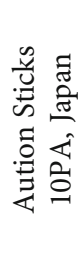 & 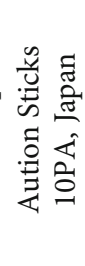 & 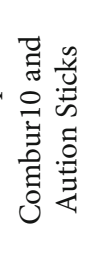 & 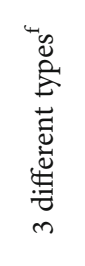 & $\overleftrightarrow{Z}$ & 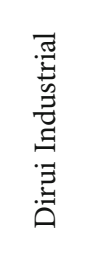 & 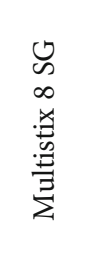 & 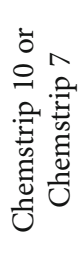 & 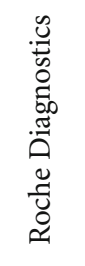 & 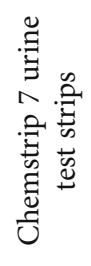 & 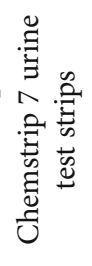 & 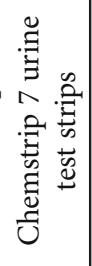 & 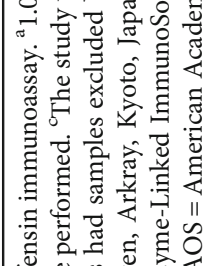 \\
\hline 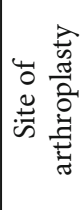 & $\overleftrightarrow{z}$ & 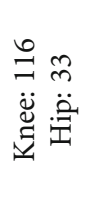 & 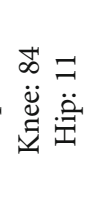 & 害 & 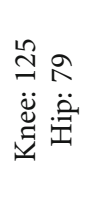 & 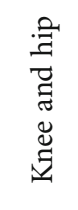 & 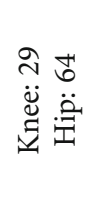 & 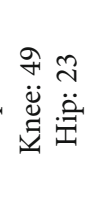 & 苂 & 古 & 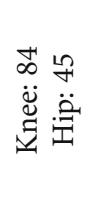 & 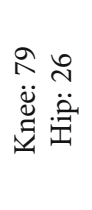 & 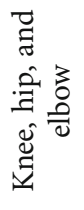 & 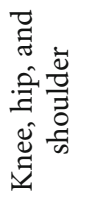 & 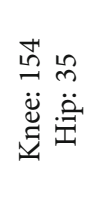 & 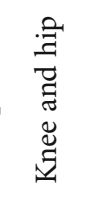 & $\underset{\Xi}{\Xi}$ & 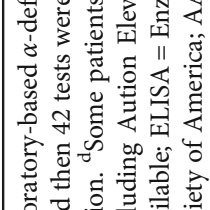 \\
\hline 逽 & $\simeq \frac{5}{0}$ & $\simeq \frac{5}{0}$ & $\simeq \frac{7}{0}$ & $\simeq \frac{\overline{0}}{0}$ & $2 \frac{\pi}{0}$ & $2+\frac{5}{0}$ & $2+\frac{\pi}{0}$ & $a \frac{\overline{0}}{0}$ & $2 \frac{ \pm}{0}$ & $\sim \frac{\pi}{0}$ & $2 \frac{\overline{0}}{0}$ & $\simeq \frac{\overline{0}}{0}$ & a $\frac{\overrightarrow{0}}{0}$ & $n=\frac{\hbar}{0}$ & $\infty \frac{7}{0}$ & $=\frac{\overline{0}}{0}$ & $=\frac{\square}{0}$ & 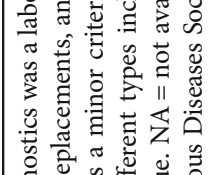 \\
\hline 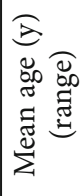 & $\overleftrightarrow{z}$ & 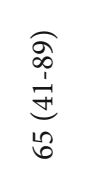 & $\begin{array}{l}\widehat{a} \\
\infty \\
\vec{J} \\
\hat{\sigma}\end{array}$ & 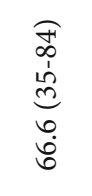 & $\tilde{6}$ & 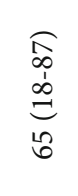 & 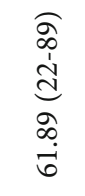 & $\stackrel{m}{0}$ & 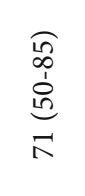 & 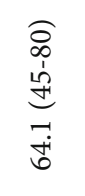 & 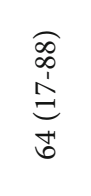 & $\overleftrightarrow{z}$ & $\begin{array}{l}\frac{a}{a} \\
\frac{i}{a} \\
\frac{-}{b}\end{array}$ & 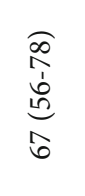 & $\begin{array}{l}\widehat{a} \\
\stackrel{a}{d} \\
\text { ñ }\end{array}$ & 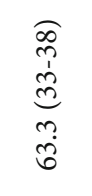 & 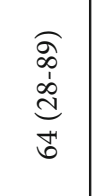 & 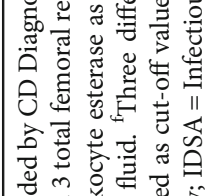 \\
\hline 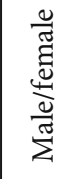 & $\overleftrightarrow{z}$ & 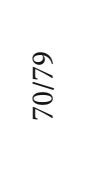 & $\stackrel{\vec{n}}{\stackrel{f}{f}}$ & 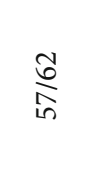 & $\underset{\infty}{\stackrel{\Xi}{\leftrightarrows}}$ & 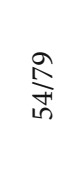 & 突 & $\stackrel{\hat{m}}{\stackrel{m}{m}}$ & $\stackrel{\text { f }}{\stackrel{9}{-~}}$ & 旁 & $\frac{8}{8}$ & 落 & $\overleftrightarrow{Z}$ & $\overleftrightarrow{z}$ & ڤे & 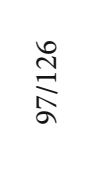 & $\frac{8}{\infty}$ & 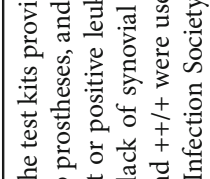 \\
\hline 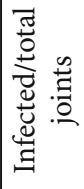 & $\stackrel{5}{2}$ & 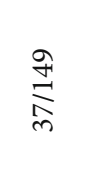 & $\frac{n}{\hat{a}}$ & 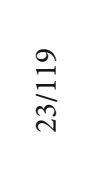 & $\underset{\substack{+\infty}}{\stackrel{+}{N}}$ & 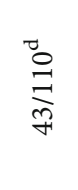 & $\underset{\substack{\infty \\
\infty}}{\infty}$ & 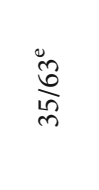 & $\frac{8}{\infty}$ & $\frac{\overrightarrow{0}}{i n}$ & 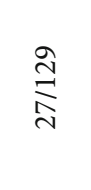 & 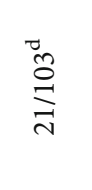 & $\frac{\sqrt{n}}{2}$ & 蒿 & $\underset{\text { in }}{\stackrel{\infty}{\gtrless}}$ & $\underset{\substack{\infty \\
\infty}}{\stackrel{n}{\infty}}$ & $\stackrel{\infty}{\stackrel{\infty}{\circ}}$ & 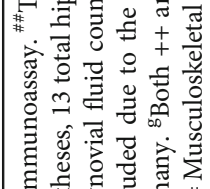 \\
\hline 吾 & 岕 & 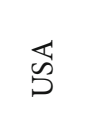 & 崩 & 氞 & 节 & .ేّ & 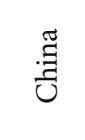 & 节 & $\begin{array}{l}\mathscr{J} \\
\stackrel{\tilde{g}}{2}\end{array}$ & 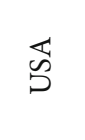 & 氞 & 光 & 点 & 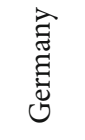 & 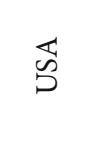 & 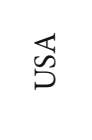 & $\begin{array}{l}\overleftrightarrow{n} \\
\text { D. }\end{array}$ & 0 \\
\hline 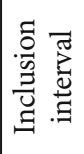 & 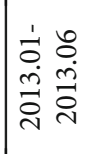 & 岁 & $\overleftrightarrow{z}$ & 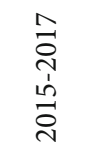 & $\begin{array}{l}0 \\
\stackrel{1}{1} \\
\stackrel{i}{+} \\
\stackrel{i}{1}\end{array}$ & 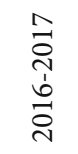 & 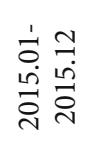 & 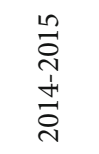 & $\begin{array}{l}n \\
\stackrel{0}{0} \\
\stackrel{1}{2} \\
\stackrel{\sim}{5}\end{array}$ & 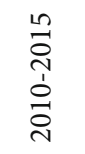 & $\begin{array}{l}n \\
\stackrel{n}{0} \\
\text { I } \\
\text { İ }\end{array}$ & $\begin{array}{l}m \\
\stackrel{0}{1} \\
\stackrel{1}{1} \\
\stackrel{\sim}{\grave{1}}\end{array}$ & 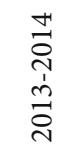 & $\overleftrightarrow{z}$ & 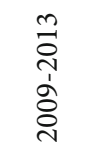 & $\overleftrightarrow{z}$ & 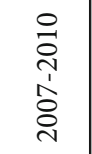 & 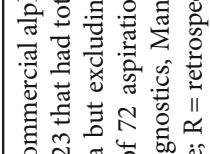 \\
\hline 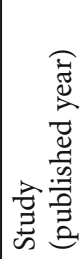 & 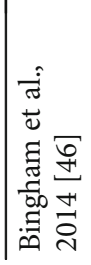 & 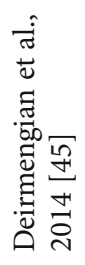 & 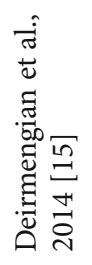 & 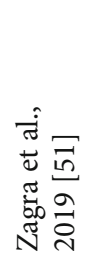 & 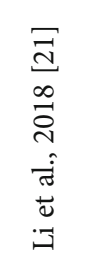 & 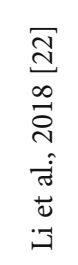 & 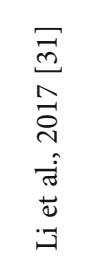 & 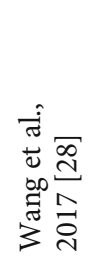 & 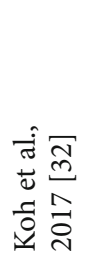 & 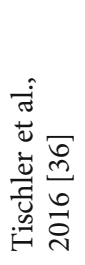 & 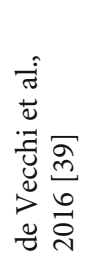 & 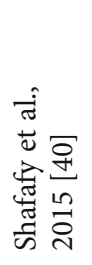 & 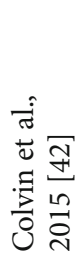 & 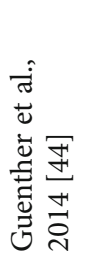 & 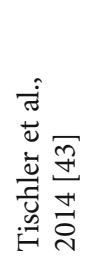 & 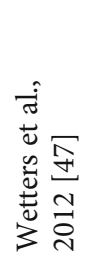 & 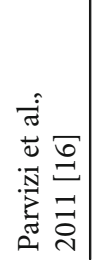 & 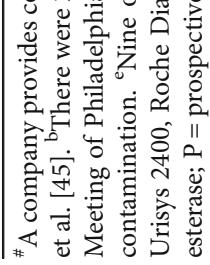 \\
\hline
\end{tabular}




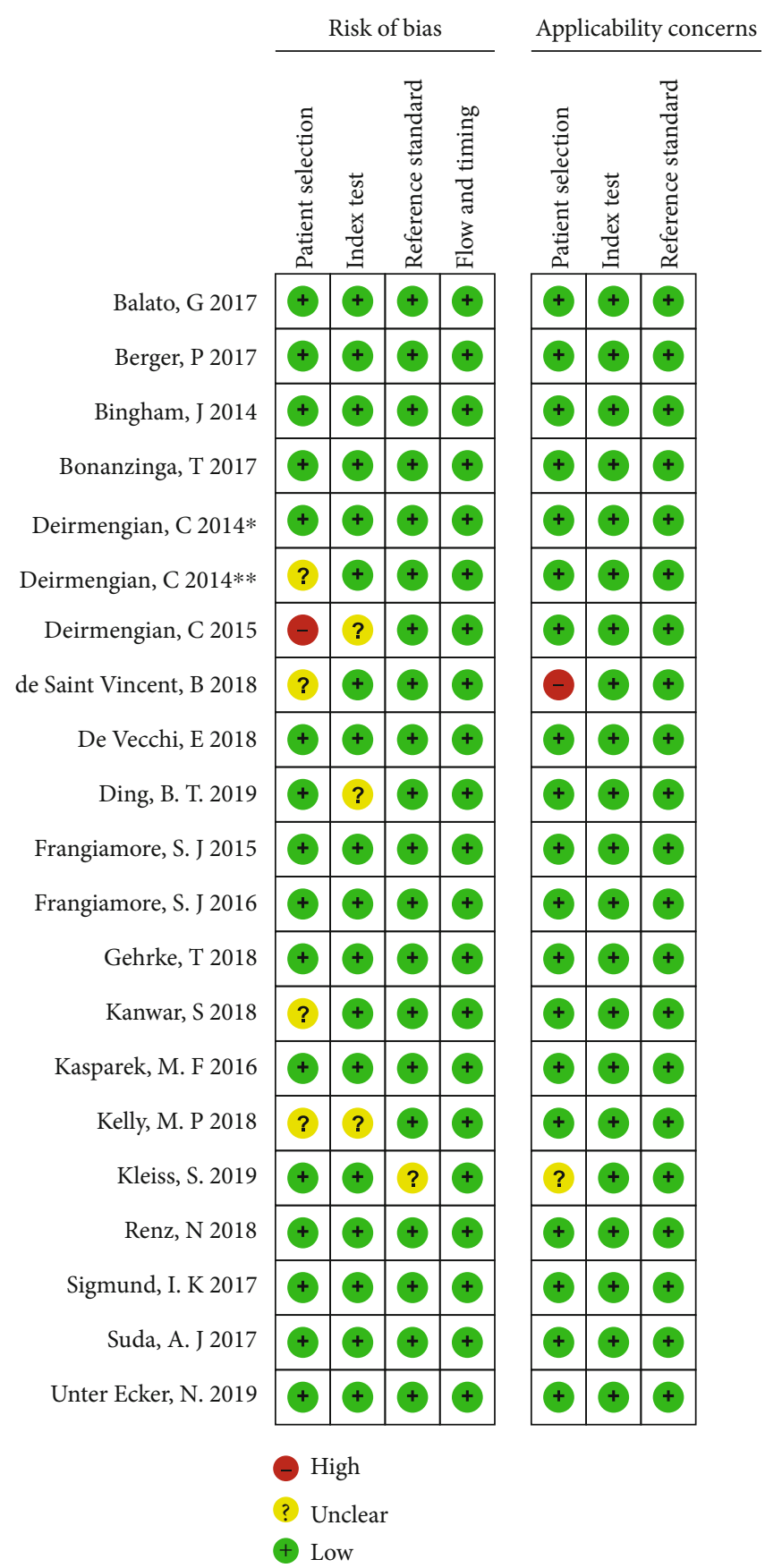

(a)

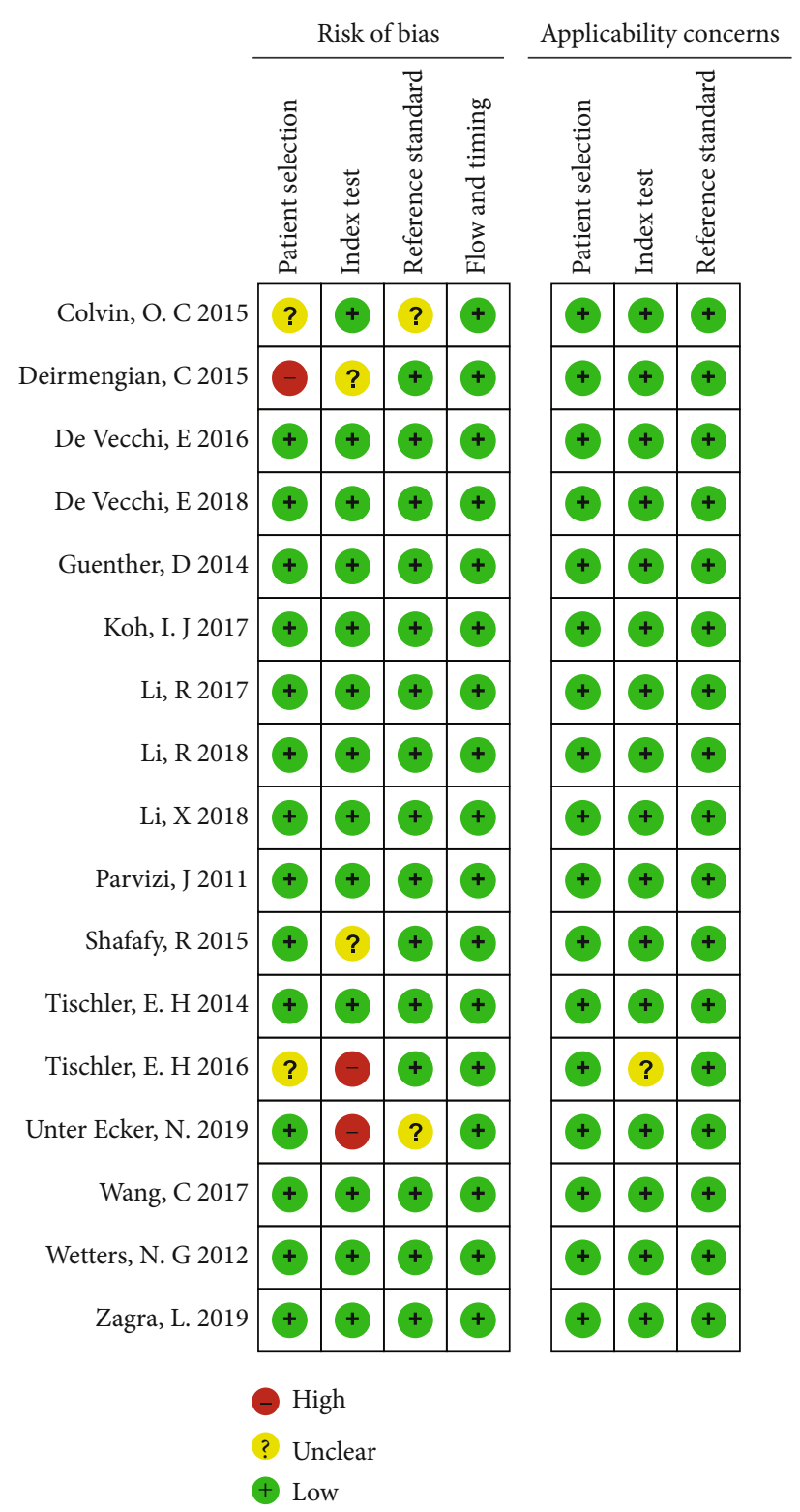

(b)

FIGURE 2: Quality assessment of included studies using QUADAS-2 tool criteria. Red in the figure indicates high risk, yellow represents unclear risk, and green means low risk.

0.98 (0.96-0.99), indicating a comparable, extremely high diagnostic ability to identify PJI using these two biomarkers.

Alpha-defensin can be detected by lateral flow test or laboratory-based alpha-defensin immunoassay. Based on the results of subgroup analysis, the diagnostic accuracy for PJI using laboratory-based alpha-defensin immunoassay was higher than that using a lateral flow test. Despite being less accurate, the lateral flow test is valuable for its high specificity, portable operation, and short operative time (responses within ten minutes). This method could be an alternative format in rapidly ruling in and, most importantly, ruling out a suspected PJI during surgery [27]. There were two studies that investigated the application of alphadefensin $[41,50]$ and one that evaluated leukocyte esterase [50] in diagnosing infection around shoulder prostheses. These reported sensitivity of $63 \%, 75 \%$, and $50 \%$, respectively, which was lower than other included studies. This might indicate that alpha-defensin and leukocyte esterase were less accurate in diagnosing PJI of the shoulder than that of the hip and knee; however, this conclusion should be 

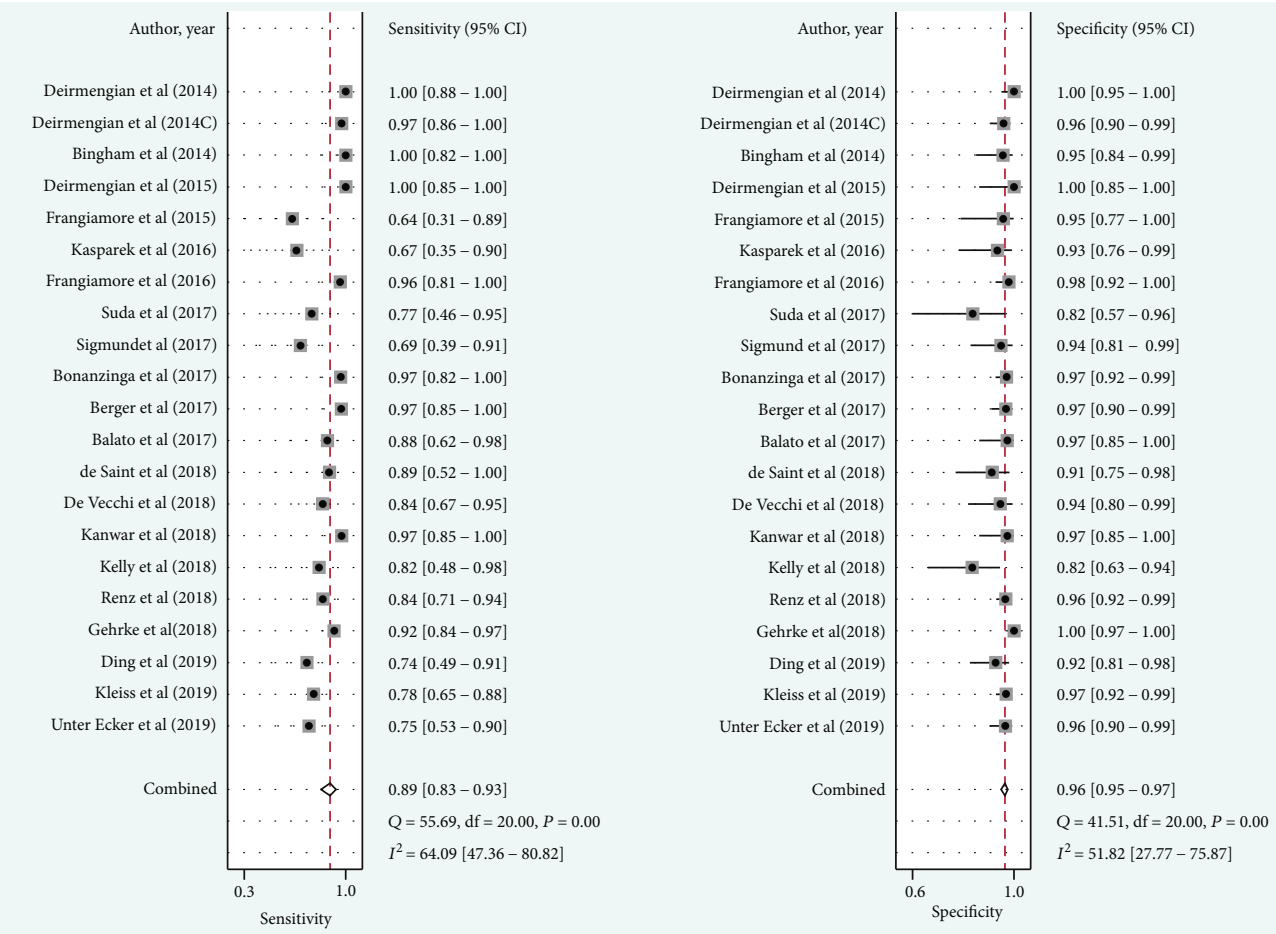

(a)

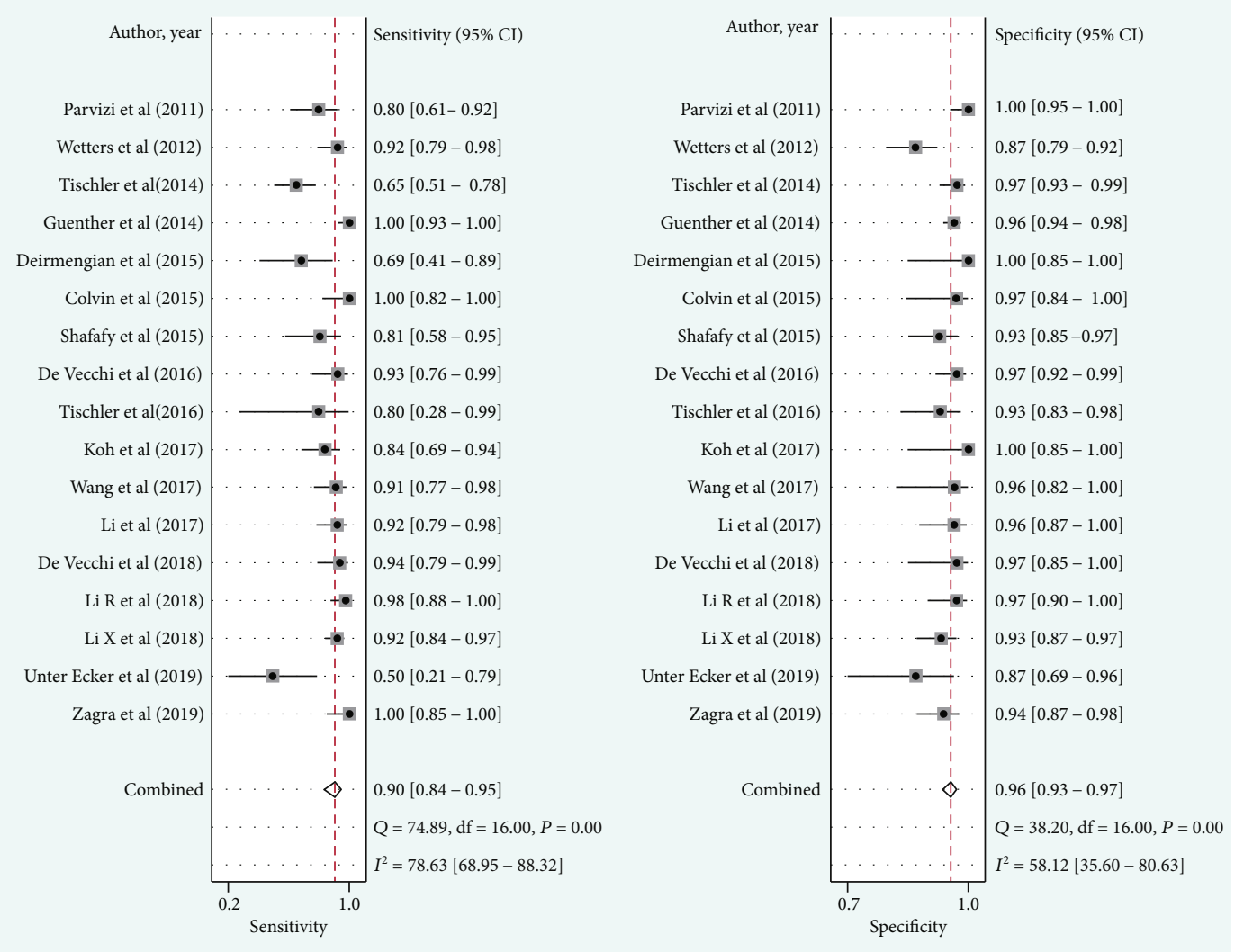

(b)

FIGURE 3: Forest plots of the sensitivity and specificity of alpha-defensin (a) and leukocyte esterase (b) for periprosthetic joint infection across all included studies. Diamonds in the central vertical lines represent pooled sensitivities or specificities with corresponding 95\% confidence interval. 


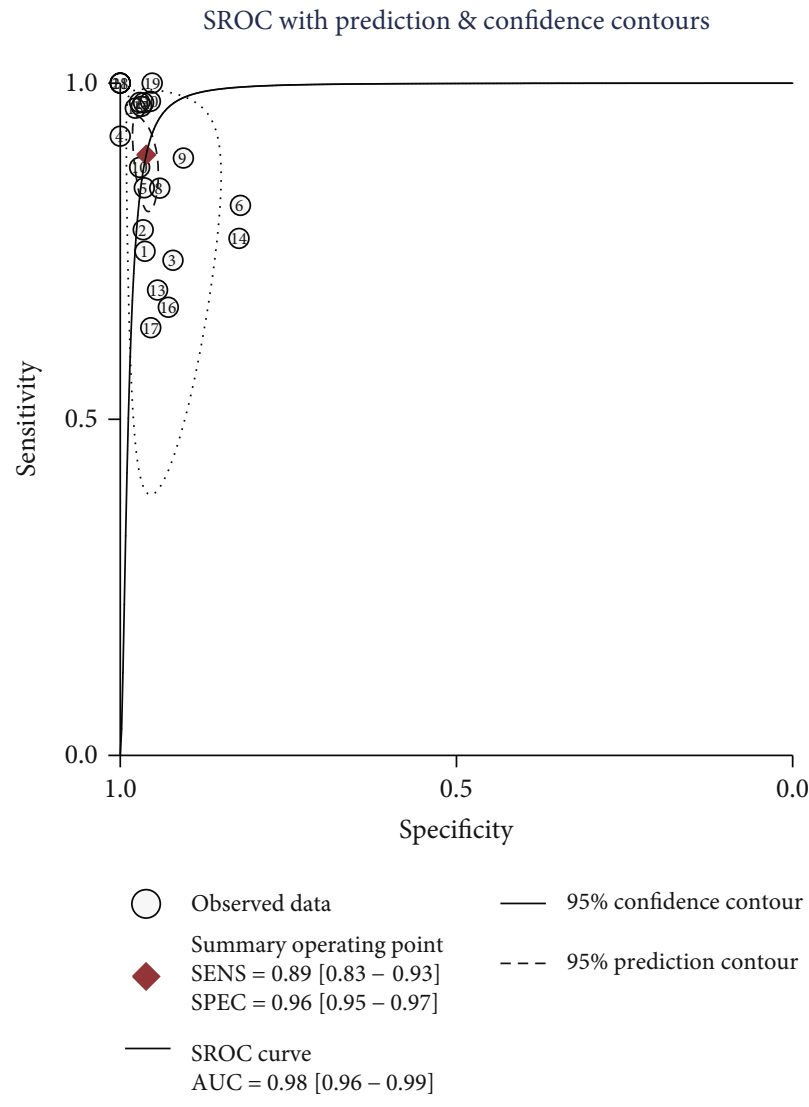

(a)

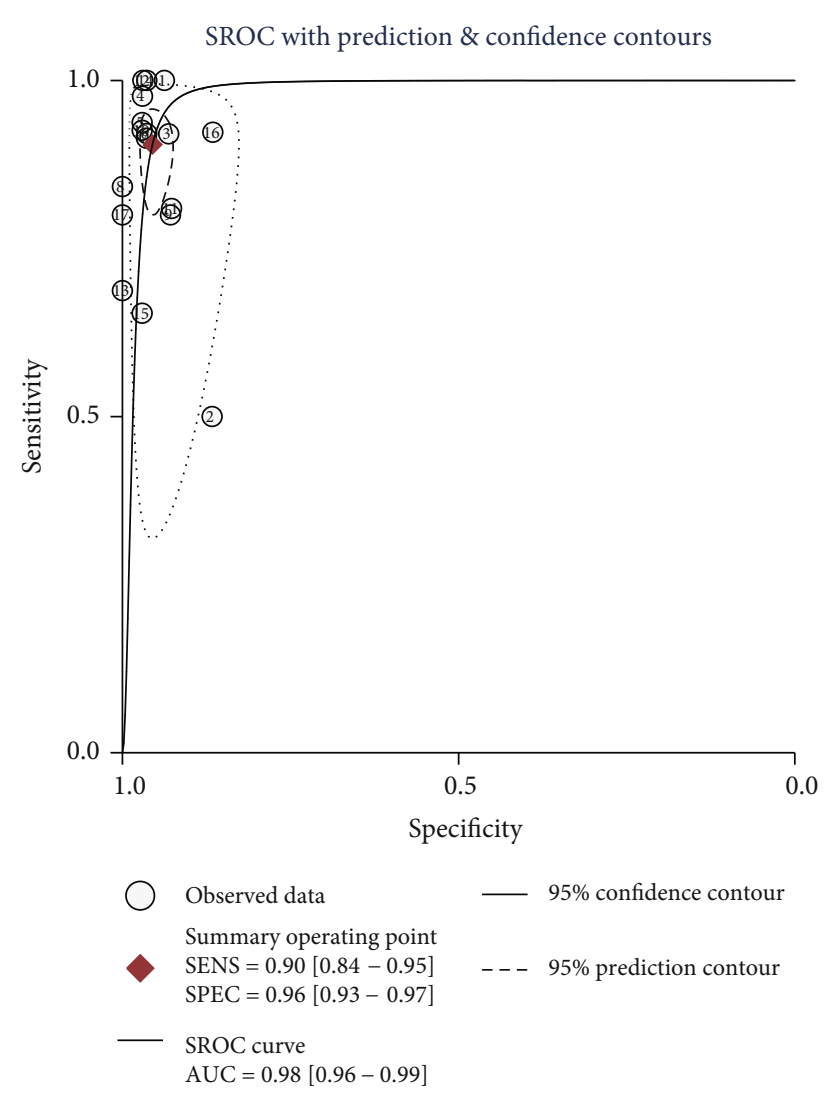

(b)

FIGURE 4: Summarized receiver operating characteristic curve (sROC) of alpha-defensin (a) and leukocyte esterase (b) for periprosthetic joint infection with corresponding $95 \%$ confidence region and the $95 \%$ prediction region.

interpreted carefully and further studies are warranted. The diagnostic accuracy of alpha-defensin seemed to be slightly better in the retrospective setting and with a larger sample size from our results of subgroup analyses. We further performed univariate metaregression analysis based on method, patient sample size, and study design and found that all factors were responsible for heterogeneity. In addition, threshold effect also accounted for the heterogeneity of studies on alpha-defensin for PJI.

Leukocyte esterase can be detected by a standard chemical test strip. Since Parvizi et al. [16] first proved that the leukocyte esterase strip could be used in the diagnosis of PJI, a standard cut-off value has remained undetermined. Based on the results of subgroup analysis, the diagnostic accuracy for PJI of the leukocyte esterase test with a "++" cut-off was much higher than that with a " $++/+$ " cut-off. Therefore, we suggest that the optimal cut-off value of leukocyte esterase in future studies or clinical applications should be "++." In addition, the subjectivity of interpretation of the result of the leukocyte esterase strip might cause a possible bias. Two studies $[16,32]$ used an automated reader instead of using the naked eye to define the final result. The sensitivities of these two studies (sensitivity $=0.80$ and 0.84 , respectively) were far below the pooled sensitivity of the current metaanalysis while the specificities of these two studies (specificity $=1.00)$ were far above the pooled specificity. We cannot conclude that it might be more accurate if the colorimetric analysis of the strip is performed by an automated reader, and further studies are warranted to evaluate the accuracy of an automated reader to define the final result of the test strip. In addition, the diagnostic accuracy of leukocyte esterase seemed to be slightly better in the prospective setting from our results of subgroup analyses. We further performed univariate metaregression analysis for cut-off, patient sample size, and study design and found that all factors were responsible for heterogeneity.

The test for alpha-defensin is simple, standardized, and validated to provide uniform results for all surgeons in clinical practice. The laboratory-based alpha-defensin immunoassay has been demonstrated to have the highest accuracy ever reported but requires more time for a response [52]. The novel lateral flow device is an alternative handy format, which is easy to use but shows lower sensitivity [30]. Meanwhile, the routine detection of alpha-defensin is much more expensive ( $\$ 760$ per test) than that for leukocyte esterase (\$0.17 per test). The advantage of leukocyte esterase for PJI diagnosis is that the method of testing is convenient and quick, returning a highly accurate result within one to two minutes, but the disadvantage is that subjective opinions might exist and samples contaminated with blood might interfere with the reading result [28]. When a patient shows any signs of infection after total knee or hip arthroplasty, 


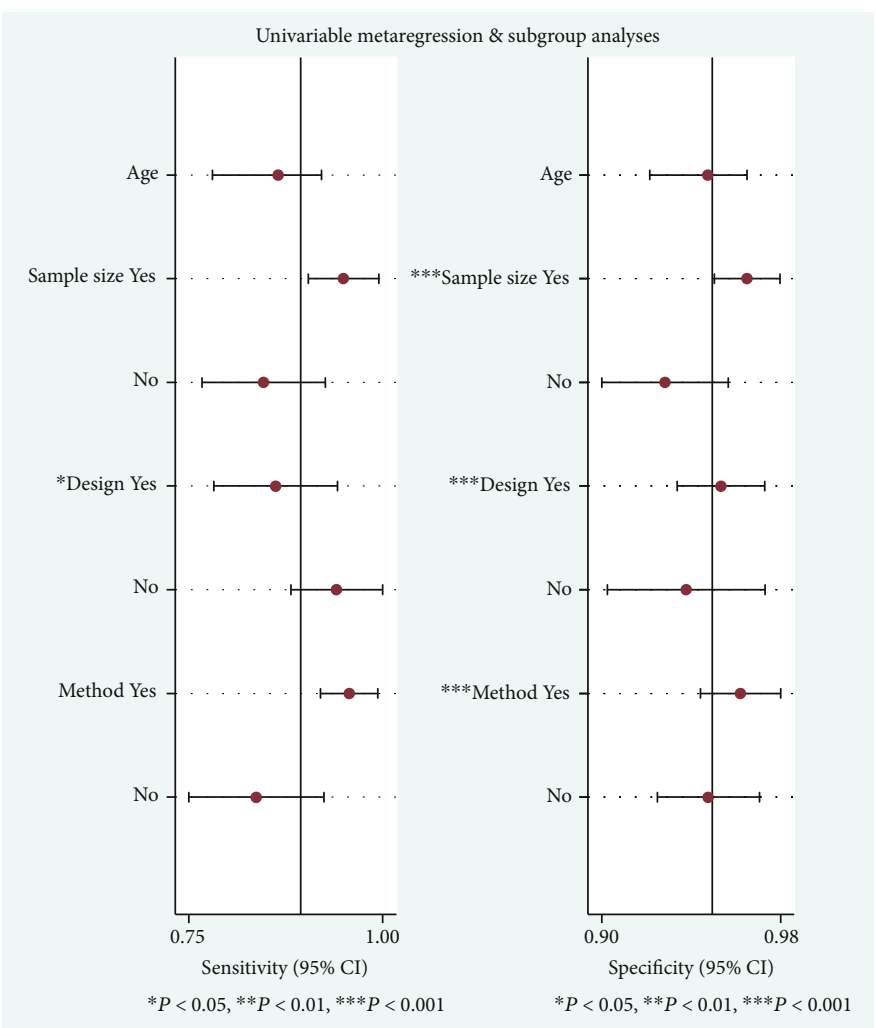

(a)

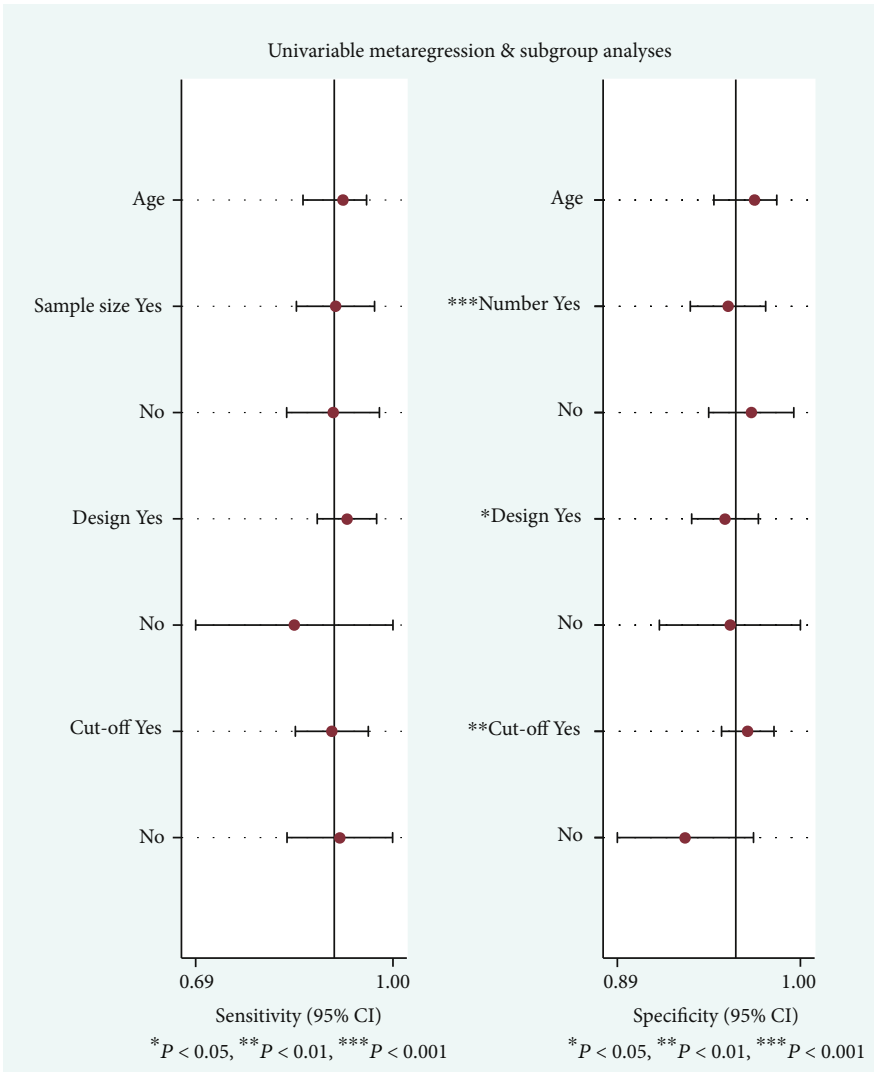

(b)

Figure 5: Graphical display of the results of univariable metaregressions of alpha-defensin (a) and leukocyte esterase (b). 


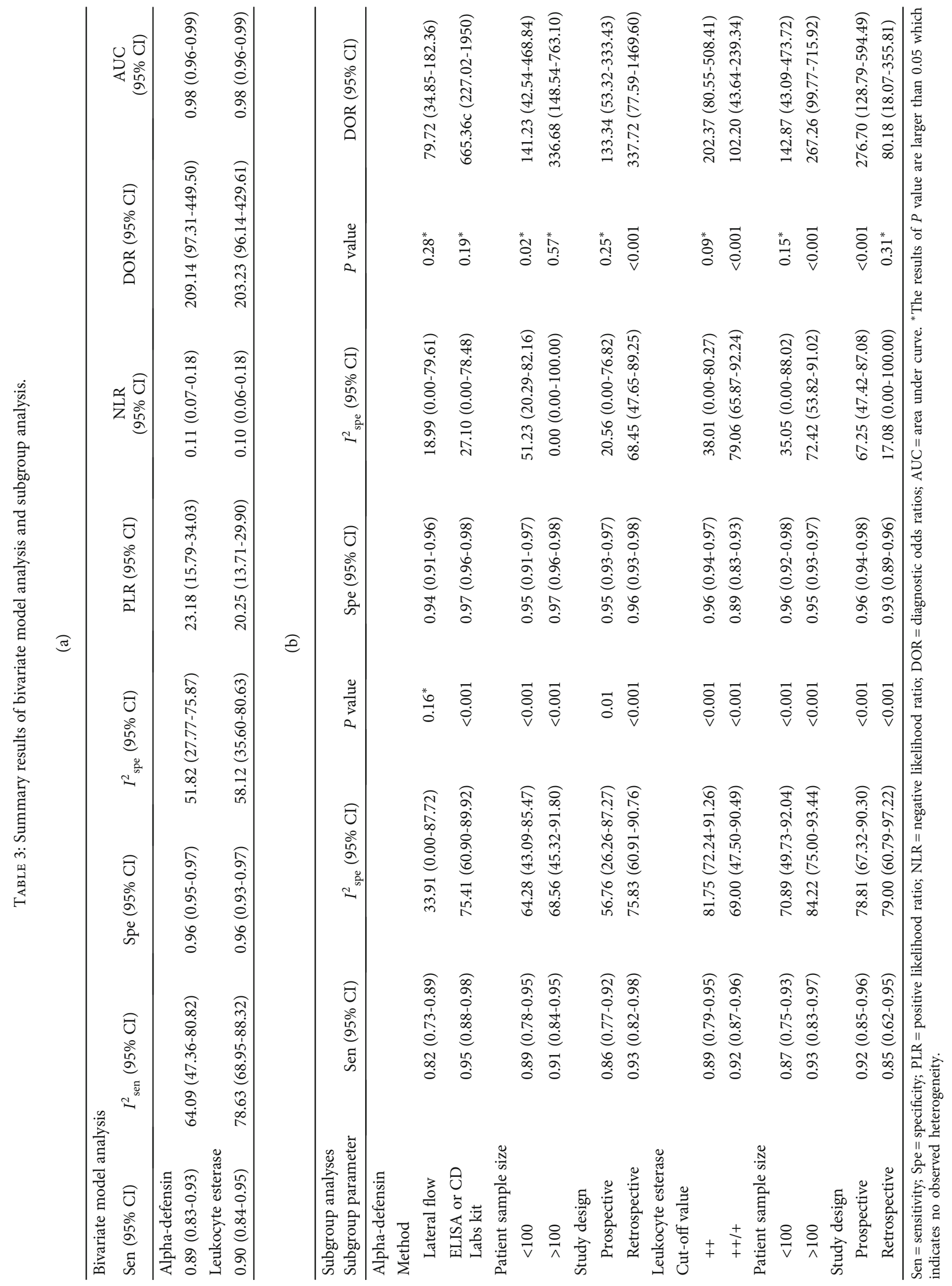


such as fever, swelling, or an elevated level of ESR or CRP, it is necessary to confirm a diagnosis of PJI and regardless of age, the leukocyte esterase test or alpha-defensin test should be carried out for both men and women. Both alphadefensin and leukocyte esterase are excellent synovial fluid markers with comparable accuracy; the leukocyte esterase test is recommended as the first resort, because the alphadefensin test is more expensive.

The strengths of the current study lie in the following two aspects. First, 31 articles were included and to our knowledge, this is the largest meta-analysis on this topic. Additionally, subgroup and metaregression analyses were conducted, which enabled us to analyze the extracted data from multiple perspectives and investigate the source of heterogeneity among the included studies.

Potential limitations of this meta-analysis should also be considered. First, three studies of alpha-defensin [14, $15,45]$ were reported by the same research group which instructed a commercial company to perform the alphadefensin test; there might therefore have been a conflict of interest. In addition, sensitivities of these three studies $[14,15,45]$ were evidently higher than those of the other included studies, which might be the reason why the pooled sensitivity of Wyatt's meta-analysis was much higher than that in our study.

\section{Conclusions}

Based on the results of the current meta-analysis, we conclude that alpha-defensin and leukocyte esterase are both excellent synovial fluid markers for diagnosing periprosthetic joint infection, with comparable and extremely high accuracy. Detection of alpha-defensin and leukocyte esterase are very convenient and could be performed preoperatively or intraoperatively.

\section{Data Availability}

The datasets used and/or analyzed during the present study are available from the corresponding author on reasonable request.

\section{Conflicts of Interest}

The authors declare that they have no competing interests.

\section{Authors' Contributions}

SW contributed to the conception of the study; LZZ and ZQY contributed significantly to literature search, data extraction, quality assessment, data analyses, and manuscript preparation; SLJ contributed to improving the article for language and style and protocol preparation; GFQ helped perform the analysis with constructive discussions; SW and LZR revised the manuscript and approved the final version. Zhizhuo Li and Qingyu Zhang are joint first authors.

\section{Acknowledgments}

This study was supported by the Beijing Natural Science Foundation (7174346 and 7182146), the National Natural Science Foundation of China (81672236, 81802224, and 81871830 ), and the Graduate Innovation Foundation of Peking Union Medical College (2019-1002-91).

\section{References}

[1] D. S. Yokoe, T. R. Avery, R. Platt, and S. S. Huang, "Reporting surgical site infections following total hip and knee arthroplasty: impact of limiting surveillance to the operative hospital," Clinical Infectious Diseases, vol. 57, no. 9, pp. 1282-1288, 2013.

[2] C. K. Koh, I. Zeng, S. Ravi, M. Zhu, K. G. Vince, and S. W. Young, "Periprosthetic joint infection is the main cause of failure for modern knee arthroplasty: an analysis of 11, 134 knees," Clinical Orthopaedics and Related Research ${ }^{\circledR}$, vol. 475, no. 9, pp. 2194-2201, 2017.

[3] S. Kurtz, K. Ong, E. Lau, F. Mowat, and M. Halpern, "Projections of primary and revision hip and knee arthroplasty in the United States from 2005 to 2030," Journal of bone and joint surgery American and British volumes (CD-ROM), vol. 89, no. 4, pp. 780-785, 2007.

[4] T. N. Peel, M. M. Dowsey, K. L. Buising, D. Liew, and P. F. M. Choong, "Cost analysis of debridement and retention for management of prosthetic joint infection," Clinical Microbiology And Infection, vol. 19, no. 2, pp. 181-186, 2013.

[5] C. E. Marculescu, T. Mabry, and E. F. Berbari, "Prevention of surgical site infections in joint replacement surgery," Surgical Infections, vol. 17, no. 2, pp. 152-157, 2016.

[6] A. J. Tande and R. Patel, "Prosthetic joint infection," Clinical Microbiology Reviews, vol. 27, no. 2, pp. 302-345, 2014.

[7] J. Parvizi and C. J. Della Valle, "AAOS clinical practice guideline diagnosis and treatment of periprosthetic joint infections of the hip and knee," Journal Of the American Academy Of Orthopaedic Surgeons, vol. 18, no. 12, pp. 760-770, 2010.

[8] D. R. Osmon, E. F. Berbari, A. R. Berendt et al., "Executive summary: diagnosis and management of prosthetic joint infection: clinical practice guidelines by the Infectious Diseases Society of America," Clinical Infectious Diseases, vol. 56, no. 1, pp. e1-e25, 2013.

[9] J. Parvizi, B. Zmistowski, E. F. Berbari et al., "New definition for periprosthetic joint infection: from the workgroup of the musculoskeletal infection society," Clinical Orthopaedics and Related Research ${ }^{\circledR}$, vol. 469, no. 11, pp. 2992-2994, 2011.

[10] G. Wang, B. Mishra, K. Lau, T. Lushnikova, R. Golla, and X. Wang, "Antimicrobial peptides in 2014," Pharmaceuticals, vol. 8, no. 1, pp. 123-150, 2015.

[11] W. D. Scheer, "The detection of leukocyte esterase activity in urine with a new reagent strip," American Journal of Clinical Pathology, vol. 87, no. 1, pp. 86-93, 1987.

[12] N. Renz, K. Yermak, C. Perka, and A. Trampuz, "Alpha defensin lateral flow test for diagnosis of periprosthetic joint infection: not a screening but a confirmatory test," The Journal of Bone and Joint Surgery, vol. 100, no. 9, pp. 742-750, 2018.

[13] K. Suen, M. Keeka, R. Ailabouni, and P. Tran, "Synovasure 'quick test' is not as accurate as the laboratory-based $\alpha$-defensin immunoassay: a systematic review and meta-analysis," Bone and Joint Journal, vol. 100-B, no. 1, pp. 66-72, 2018. 
[14] C. Deirmengian, K. Kardos, P. Kilmartin et al., "The alphadefensin test for periprosthetic joint infection outperforms the leukocyte esterase test strip," Clinical Orthopaedics and Related Research ${ }^{\circledast}$, vol. 473, no. 1, pp. 198-203, 2015.

[15] C. Deirmengian, K. Kardos, P. Kilmartin, A. Cameron, K. Schiller, and J. Parvizi, "Diagnosing periprosthetic joint infection: has the era of the biomarker arrived?" Clinical Orthopaedics and Related Research ${ }^{\circledR}$, vol. 472, no. 11, pp. 3254-3262, 2014.

[16] J. Parvizi, C. Jacovides, V. Antoci, and E. Ghanem, "Diagnosis of periprosthetic joint infection: the utility of a simple yet unappreciated enzyme," The Journal of Bone \& Joint Surgery, vol. 93, no. 24, pp. 2242-2248, 2011.

[17] M. C. Wyatt, A. D. Beswick, S. K. Kunutsor, M. J. Wilson, M. R. Whitehouse, and A. W. Blom, "The alpha-defensin immunoassay and leukocyte esterase colorimetric strip test for the diagnosis of periprosthetic infection: a systematic review and meta-analysis," The Journal of bone and joint surgery. American volume, vol. 98, no. 12, pp. 992-1000, 2016.

[18] M. M. G. Leeflang, J. J. Deeks, C. Gatsonis, P. M. M. Bossuyt, and Cochrane Diagnostic Test Accuracy Working Group, "Systematic reviews of diagnostic test accuracy," Annals of internal medicine, vol. 149, no. 12, pp. 889-897, 2008.

[19] D. Moher, A. Liberati, J. Tetzlaff, D. G. Altman, and PRISMA Group, "Preferred reporting items for systematic reviews and meta-analyses: the PRISMA statement," Journal of Clinical Epidemiology, vol. 62, no. 10, pp. 1006-1012, 2009.

[20] P. F. Whiting, A. W. S. Rutjes, M. E. Westwood et al., "QUADAS-2: a revised tool for the quality assessment of diagnostic accuracy studies," Annals of Internal Medicine, vol. 155, no. 8, pp. 529-536, 2011.

[21] X. Li, R. Li, M. Ni et al., "Leukocyte esterase strip test: a rapid and reliable method for the diagnosis of infections in arthroplasty," Orthopedics, vol. 41, no. 2, pp. e189-e193, 2018.

[22] R. Li, Q. Lu, Y. G. Zhou, W. Chai, S. B. Lu, and J. Y. Chen, "Centrifugation may change the results of leukocyte esterase strip testing in the diagnosis of periprosthetic joint infection," The Journal of arthroplasty, vol. 33, no. 9, pp. 2981-2985, 2018.

[23] M. P. Kelly, B. Darrith, C. P. Hannon, D. Nam, P. M. Courtney, and C. J. Della Valle, "Synovial fluid alpha-defensin is an adjunctive tool in the equivocal diagnosis of periprosthetic joint infection," The Journal of Arthroplasty, vol. 33, no. 11, pp. 3537-3540, 2018.

[24] S. Kanwar, A. A. Al-Mansoori, M. R. Chand, J. M. Villa, J. C. Suarez, and P. D. Patel, "What is the optimal criteria to use for detecting periprosthetic joint infections before total joint arthroplasty?," The Journal of Arthroplasty, vol. 33, no. 7, pp. S201-S204, 2018.

[25] T. Gehrke, C. Lausmann, M. Citak, T. Bonanzinga, L. Frommelt, and A. Zahar, "The accuracy of the alpha defensin lateral flow device for diagnosis of periprosthetic joint infection: comparison with a gold standard," The Journal of Bone and Joint Surgery, vol. 100, no. 1, pp. 42-48, 2018.

[26] E. De Vecchi, C. L. Romanò, R. De Grandi, L. Cappelletti, F. Villa, and L. Drago, "Alpha defensin, leukocyte esterase, C-reactive protein, and leukocyte count in synovial fluid for pre-operative diagnosis of periprosthetic infection," International Journal of Immunopathology and Pharmacology, vol. 32, 2018.

[27] B. de Saint Vincent, H. Migaud, E. Senneville et al., "Diagnostic accuracy of the alpha defensin lateral flow device (Synovasure) for periprosthetic infections in microbiologically complex situ- ations: a study of 42 cases in a French referral centre," Orthopaedics \& Traumatology: Surgery \& Research, vol. 104, no. 4, pp. 427-431, 2018.

[28] C. Wang, R. Li, Q. Wang, J. Duan, and C. Wang, "Leukocyte esterase as a biomarker in the diagnosis of periprosthetic joint infection," Medical Science Monitor, vol. 23, pp. 353-358, 2017.

[29] A. J. Suda, M. Tinelli, N. D. Beisemann, Y. Weil, A. Khoury, and O. E. Bischel, "Diagnosis of periprosthetic joint infection using alpha-defensin test or multiplex-PCR: ideal diagnostic test still not found," International Orthopaedics, vol. 41, no. 7, pp. 1307-1313, 2017.

[30] I. K. Sigmund, J. Holinka, J. Gamper et al., "Qualitative $\alpha$ defensin test (Synovasure) for the diagnosis of periprosthetic infection in revision total joint arthroplasty," The Bone \& Joint Journal, vol. 99-B, no. 1, pp. 66-72, 2017.

[31] R. Li, X. Li, B. Yu et al., "Comparison of leukocyte esterase testing of synovial fluid with synovial histology for the diagnosis of periprosthetic joint infection," Medical Science Monitor, vol. 23, pp. 4440-4446, 2017.

[32] I. J. Koh, S. B. Han, Y. in et al., "The leukocyte esterase strip test has practical value for diagnosing periprosthetic joint infection after total knee arthroplasty: a multicenter study," The Journal of Arthroplasty, vol. 32, no. 11, pp. 3519-3523, 2017.

[33] T. Bonanzinga, A. Zahar, M. Dütsch, C. Lausmann, D. Kendoff, and T. Gehrke, "How reliable is the alphadefensin immunoassay test for diagnosing periprosthetic joint infection? A prospective study," Clinical Orthopaedics and Related Research ${ }^{\circledR}$, vol. 475, no. 2, pp. 408-415, 2017.

[34] P. Berger, M. Van Cauter, R. Driesen, J. Neyt, O. Cornu, and J. Bellemans, "Diagnosis of prosthetic joint infection with alpha-defensin using a lateral flow device," The bone \& joint journal, vol. 99-b, no. 9, pp. 1176-1182, 2017.

[35] G. Balato, V. Franceschini, T. Ascione et al., "High performance of $\alpha$-defensin lateral flow assay (Synovasure) in the diagnosis of chronic knee prosthetic infections," Knee Surgery, Sports Traumatology, Arthroscopy, vol. 26, no. 6, pp. 1717-1722, 2018.

[36] E. H. Tischler, D. R. Plummer, A. F. Chen, C. J. Della Valle, and J. Parvizi, "Leukocyte esterase: metal-on-metal failure and periprosthetic joint infection," The Journal of Arthroplasty, vol. 31, no. 10, pp. 2260-2263, 2016.

[37] M. F. Kasparek, M. Kasparek, F. Boettner, M. Faschingbauer, J. Hahne, and M. Dominkus, "Intraoperative diagnosis of periprosthetic joint infection using a novel alpha-defensin lateral flow assay," The Journal of Arthroplasty, vol. 31, no. 12, pp. 2871-2874, 2016.

[38] S. J. Frangiamore, N. D. Gajewski, A. Saleh, M. Farias-Kovac, W. K. Barsoum, and C. A. Higuera, " $\alpha$-Defensin accuracy to diagnose periprosthetic joint infection-best available test?," The Journal of Arthroplasty, vol. 31, no. 2, pp. 456-460, 2016.

[39] E. de Vecchi, F. Villa, M. Bortolin et al., "Leucocyte esterase, glucose and C-reactive protein in the diagnosis of prosthetic joint infections: a prospective study," Clinical Microbiology and Infection, vol. 22, no. 6, pp. 555-560, 2016.

[40] R. Shafafy, W. McClatchie, K. Chettiar et al., "Use of leucocyte esterase reagent strips in the diagnosis or exclusion of prosthetic joint infection," The bone \& joint journal, vol. 97-b, no. 9, pp. 1232-1236, 2015.

[41] S. J. Frangiamore, A. Saleh, M. J. Grosso et al., " $\alpha$-Defensin as a predictor of periprosthetic shoulder infection," Journal of Shoulder and Elbow Surgery, vol. 24, no. 7, pp. 1021-1027, 2015. 
[42] O. C. Colvin, M. J. Kransdorf, C. C. Roberts et al., "Leukocyte esterase analysis in the diagnosis of joint infection: can we make a diagnosis using a simple urine dipstick?," Skeletal Radiology, vol. 44, no. 5, pp. 673-677, 2015.

[43] E. H. Tischler, P. K. Cavanaugh, and J. Parvizi, "Leukocyte esterase strip test: matched for musculoskeletal infection society criteria," The Journal of Bone and Joint Surgery, vol. 96, no. 22, pp. 1917-1920, 2014.

[44] D. Guenther, T. Kokenge, O. Jacobs et al., "Excluding infections in arthroplasty using leucocyte esterase test," International Orthopaedics, vol. 38, no. 11, pp. 2385-2390, 2014.

[45] C. Deirmengian, K. Kardos, P. Kilmartin, A. Cameron, K. Schiller, and J. Parvizi, "Combined measurement of synovial fluid $\alpha$-defensin and C-reactive protein levels: highly accurate for diagnosing periprosthetic joint infection," The Journal of Bone and Joint Surgery, vol. 96, no. 17, pp. 1439-1445, 2014.

[46] J. Bingham, H. Clarke, M. Spangehl, A. Schwartz, C. Beauchamp, and B. Goldberg, "The alpha defensin-1 biomarker assay can be used to evaluate the potentially infected total joint arthroplasty," Clinical Orthopaedics and Related Research $^{\circledR}$, vol. 472, no. 12, pp. 4006-4009, 2014.

[47] N. G. Wetters, K. R. Berend, A. V. Lombardi, M. J. Morris, T. L. Tucker, and C. J. Della Valle, "Leukocyte esterase reagent strips for the rapid diagnosis of periprosthetic joint infection," The Journal of Arthroplasty, vol. 27, no. 8, pp. 8-11, 2012.

[48] B. T. Ding, K. G. Tan, C. Y. Kau, H. Y. H. Chan, and M. F. B. Mohd Fadil, "Accuracy of the $\alpha$-defensin lateral flow assay for diagnosing periprosthetic joint infection in Asians," Journal of orthopaedic surgery, vol. 27, no. 1, 2019.

[49] S. Kleiss, N. M. Jandl, A. Novo de Oliveira, W. Rüther, and A. Niemeier, "Diagnostic accuracy of alpha-defensin enzyme-linked immunosorbent assay in the clinical evaluation of painful hip and knee arthroplasty with possible prosthetic joint infection: a prospective study of 202 cases," The bone \& joint journal, vol. 101-b, no. 8, pp. 970-977, 2019.

[50] N. Unter Ecker, A. Koniker, T. Gehrke et al., "What is the diagnostic accuracy of alpha-defensin and leukocyte esterase test in periprosthetic shoulder infection?," Clinical orthopaedics and related research, vol. 477, no. 7, pp. 1712-1718, 2019.

[51] L. Zagra, F. Villa, L. Cappelletti, E. Gallazzi, G. Materazzi, and E. De Vecchi, "Can leucocyte esterase replace frozen sections in the intraoperative diagnosis of prosthetic hip infection?," The bone \& joint journal, vol. 101-b, no. 4, pp. 372-377, 2019.

[52] T. Bonanzinga, M. C. Ferrari, G. Tanzi, F. Vandenbulcke, A. Zahar, and M. Marcacci, "The role of alpha defensin in prosthetic joint infection (PJI) diagnosis: a literature review," EFORT Open Reviews, vol. 4, no. 1, pp. 10-13, 2019. 